\title{
IRIS Recognition based on Non Linear Dimensionality Reduction of IRIS Code with KPCA and SVM based Classification
}

\author{
V. V. Satyanarayana Tallapragada \\ Assistant Professor \\ Department of E.C.E., C.B.I.T. \\ Gandipet, Hyderabad, India.
}

\author{
E. G. Rajan \\ President \\ Pentagram Research Center \\ Jubilee Hills, Hyderabad, India
}

\begin{abstract}
Iris recognition technique has come a long way since a comprehensive method was first proposed by Daugman two decades back. But with evolution of technology and processing environment, several techniques are being proposed and tested. The study clearly shows that IRIS segmentation based on the Daugman's technique and IRIS recognition based on the Gabor features extracted from the segmented IRIS images is the most efficient technique for recognition. IRIS recognition research can be understood as two point agenda: extracting better features from segmented IRIS images and use a better classifier than the Hamming distance based classification. With the increase of number of features recognition error decreases and accuracy increases, but other complexities like space complexity for storing the features and time complexity for optimizing the features by kernel based classifier is difficult. Hence in this work we emphasize on extracting the most significant feature set from the segmented IRIS and project the features in a high dimensional space using KPCA dimensionality reduction technique. The features are classified using Multiclass support vector machine. Results show that the recognition rate and FAR of the proposed technique are very high when compared to Multi Class SVM.
\end{abstract}

\section{General Terms}

IRIS recognition, Daugman's Technique, KPCA, SVM.

\section{Keywords}

Reduction of IRIS Code, KPCA and SVM.

\section{INTRODUCTION}

IRIS recognition problem may be considered as a problem of classifying the features extracted from a test iris image to one of the feature groups which are taken as training images or iris image of known classes or persons. Feature classification based IRIS recognition techniques are more popular than the hamming distance based iris recognition problem, due to better efficiency of the classifiers than the distance based recognition system. The classifiers can interpolate the features extracted from IRIS images to a high dimensional vector plane and calculate the distance from the given IRIS features with that of features already taken as training samples. It is already proved that values are more separable at the high dimensional plane than the low dimensional plane. Therefore Classifier based IRIS recognition technique is preferred over hamming distance. There are several good classifiers which can be used for classifying the features and hence recognizing IRIS. For example Neural Network, KNN classifier, SVM classifier, Neuro Fuzzy Classifiers[14] are some of the most widely used classifiers. Literature has already proved that Support vector machine[10,11] or SVM based classifiers perform better than other peers in a complex iris recognition problem with thousands of classes and hundreds of features. But one of the major limitation of kernel based classifier is the speed[9]. As the number of classes increases, the complexity of the classifier is also increased[9]. Therefore kernel based classifiers cannot be used practically unless and until the features are optimized and the speed is improved. In much literature, a dimensionality reduction technique is proposed to reduce the number of dimensions of the features extracted from the IRIS images[16]. Dimensionality reduction techniques are dependent on the correlation of the features. Therefore the test dimensions are to be reduced along with the trained features for obtaining a dimension vector which is unique to both the input and output planes. This again has the limitation that the trained vectors needs to be stored nonreduced. Therefore dimensionality reduction becomes an optimization problem which only assists the kernel based classifiers to perform faster. The core objective of this work is to device a technique to reduce the input and test dimensionality separately. We build a model based on the input dimensionality reduction and the model is applied on the test features to extract the reduced test features. Therefore the stored trained features are reduced features which solve the purpose of space complexity. This is particularly significant for classifiers that operate on network, as fetching huge number of features consumes more bandwidth.

IRIS images from a specific database (here the proposed system is tested with UBIRIS[12] and CASIA IRIS database[17]) is analyzed and the outer and the inner radius of the IRIS part is selected. IRIS part is segmented by first calculating Hough Circles over the eye image and followed by selecting those circles satisfying the radial dimensions selected first. Segmented IRIS image is further processed for removing eye yields by calculating the Hough lines and removing the highest Hough lines from the segmented IRIS part. Circular iris image is transformed to rectangular coordinate of fixed size. This image is filtered using 2 dimensional Gabor filter. The filter produces $\mathrm{M}$ x $\mathrm{N}$ non zero features where $\mathrm{M}, \mathrm{N}$ is the size of the normalized rectangular 


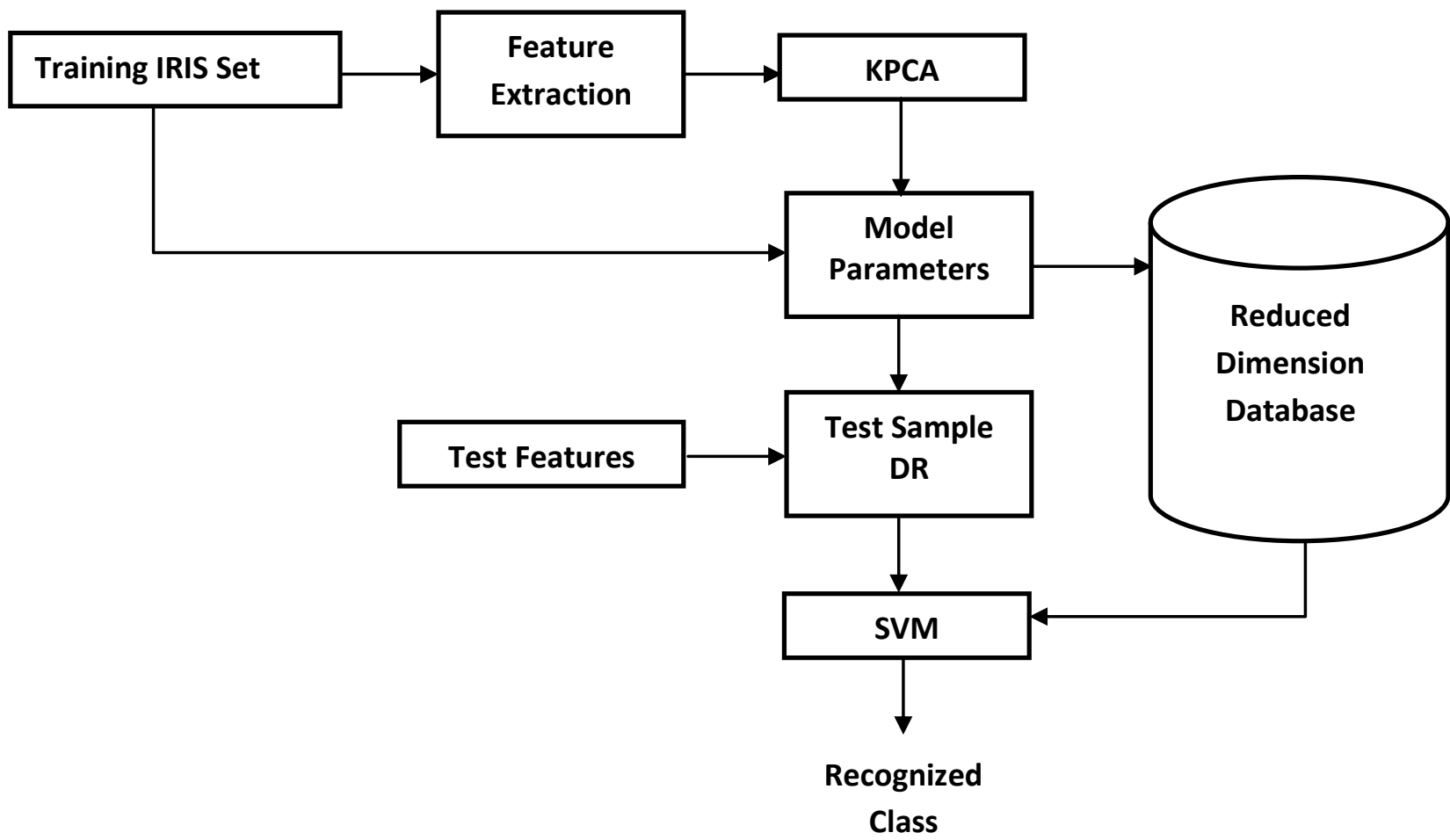

Fig 1: Block Diagram of Proposed Technique

IRIS image. These coefficients of filter output are used as high dimensional feature vector which is reduced using KPCA. The reduced features are stored in the database and SVM is used to classify the features of given test IRIS image from the trained reduced feature vectors.

\section{RELATED WORK}

The most unique observable feature in humans face is the detailed texture of iris in the eye [1]. Advances in iris recognition are: detection and modeling of the inner and outer boundaries of the iris with active contours, having an orthographic perspective by rotating the eye by the aid of trigonometry and projective geometry, statistical inference methods for detecting and excluding eyelashes, depending on the amount of iris data [2]. Till now many algorithms for recognizing the persons by their iris patterns have been developed. But the false matches increases in many cases. The recognition principle is the failure of a test of statistical independence on iris phase structure encoded by multi-scale quadrature wavelets[3]. Inorder to recognize the person in a large dataset, some features must be extracted from the trained iris image. Henceforth the selection of the optimal feature subset and the classification has become a major concern[4]. A novel iris segmentation scheme based on the chain code and collarette area localization is proposed to improve the iris recognition systems accuracy[5]. In a large dataset clustering of the same images leads to a fast recognition system. KPCA combined with KMeans and the GMM (Gaussian Mixture Model) performs well than the trandition techniques[6]. For real world data improvement in KPCA is more suitable[7]. Gabor wavelets and Kernel methods are combined in order to achieve highly discriminative features for recognition [15]. The kernel techniques are nothing but mapping low dimensional image data into a high dimensional feature space [8]. The higher the accuracy desired for patterns the higher would be dimensional plane of representation. The dimensional enhancement of the data also increases the computational complexity and space complexity which minimizes the overall efficiency of a recognition system even after accuracy improvement due to slow processing [16]

\section{METHODOLOGY}

Consider a dataset of features extracted from IRIS images of known persons or class. Let each row represents a set of features or dimensions that demonstrates a particular instance of an IRIS image. If the number of properties is huge, then the possible rows are exponentially high. Thus, the larger the dimensionality, the more difficult it becomes to sample the space. This causes many problems. Algorithms that work on high-dimensional data generally have a high time-complexity. High-dimensional data is a problem to many machine learning algorithms (MLA). This is known as the curse of dimensionality. Reducing data into fewer dimensions makes algorithms analysis more efficient, and can help MLA make more accurate predictions. While reducing the properties, the techniques take into account of the hidden or the inner dimension of the data. Therefore this is also referred to as a projection plane or high dimensional vector space.

For example segmented IRIS image of all the registered persons will have distinct veins which help identifying the IRIS images. Gabor convolve over these iris images, will return several features. Not each feature is distinct and recognition can be considered as a problem of detecting the phase of the appearance of the veins. Therefore a non linear mapping based technique can be used to extract the inner dimensionality of the IRIS images.

There are several techniques for dimensionality reduction which includes PCA based technique, SOM based techniques and so on. The proposed classifier relies on the kernel trick or mapping the features to a different data space where the values are separable, hence a kernel based reduction technique is better suited. 
As PCA gives most prominent and significant eigen vectors of any feature set, we use KPCA based technique to reduce the dimension of the feature space.

It is a combination of Principal Component Analysis and the Kernel trick. PCA begins by computing the covariance matrix of the $m \times n$ matrix $X$.

$$
C=\frac{1}{m} \sum_{i=1}^{m} x_{i} x_{i}^{T} \ldots
$$

It then projects the data onto the first $\mathrm{k}$ eigenvectors of that matrix. By comparison, KPCA begins by computing the covariance matrix of the data after being transformed into a higher-dimensional space,

$$
C=\frac{1}{m} \sum_{i=1}^{m} \Phi\left(x_{i}\right) \Phi\left(x_{i}\right)^{T} \ldots
$$

It then projects the data onto the first $\mathrm{k}$ eigenvectors of the matrix such that it incorporates only relevant information, just like PCA. It uses the kernel trick to factor away much of the computation, such that the entire process can be performed without actually computing $\Phi(x)$ as $\Phi$ must be chosen in a way that suits kernel.

KPCA is a model; therefore it can be used to map points onto its embedding that were not available at training time. Main advantage of this model is the fact that the vectors are first transformed and then mapped to eigen plane. Hence the test vectors need not to be reduced along with the trained vectors. Low dimensional data is saved as training database which is classified using multiclass SVM.

\section{RESULTS}

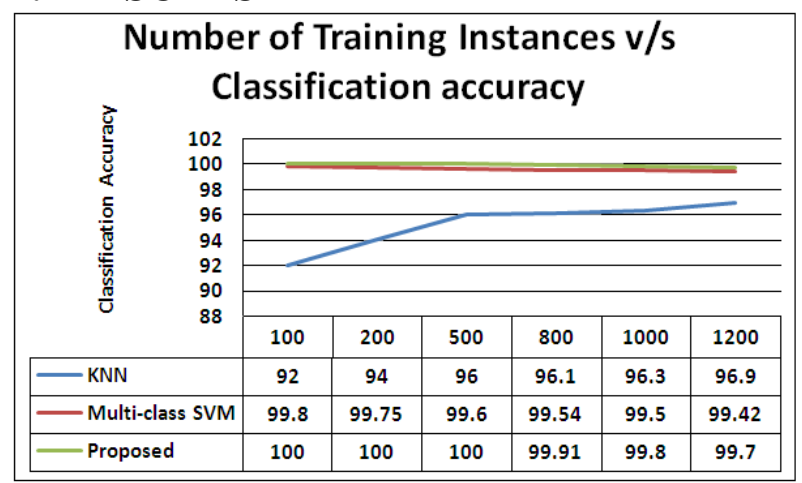

Fig 2: Plot of Number of Training Instances (number of registered persons $x$ independent iris image of each person) versus Classification Accuracy

Figure 2 shows that the recognition rate for 500 classes of the proposed technique is $100 \%$ and for 1200 classes the recognition accuracy is $99.7 \%$, with only three misdetection out of 1000 test instances. This clearly proves that with appropriate minimization of the features, recognition rate of the classifier is improved to a great deal. As kernel based classifiers like SVM depends upon separability of the vectors, the accuracy depends purely on selection and optimization of the features. Though features are optimized by SVM prior to classification, reduction of the features through a separate dimensionality reduction technique attributes to better kernel performance.

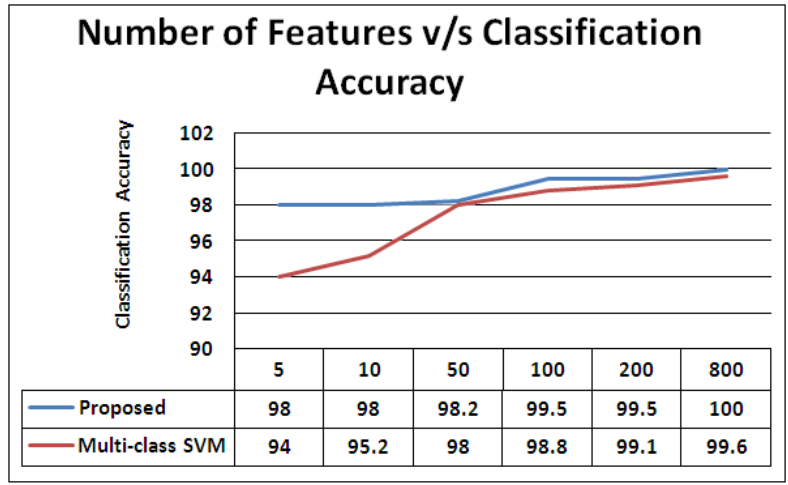

Fig 3: Plot of Number of Features versus Classification Accuracy

The graph in figure 3 shows that as number of features are increased, classification accuracy also increases which is expected as more features defines better decision boundary for the kernel based classifiers. But the interesting fact that comes out of the analysis of figure 3 is that the performance of the proposed system is quite high even for less number of features. Here number of features is selected through intrinsic dimensionality test over the available feature domain. The intrinsic dimensionality or data dependency over the entire range of training data is consistent. Therefore the classification accuracy is better a system where features are reduced to optimum by a kernel based non linear dimensionality reduction technique before the recognition stage.

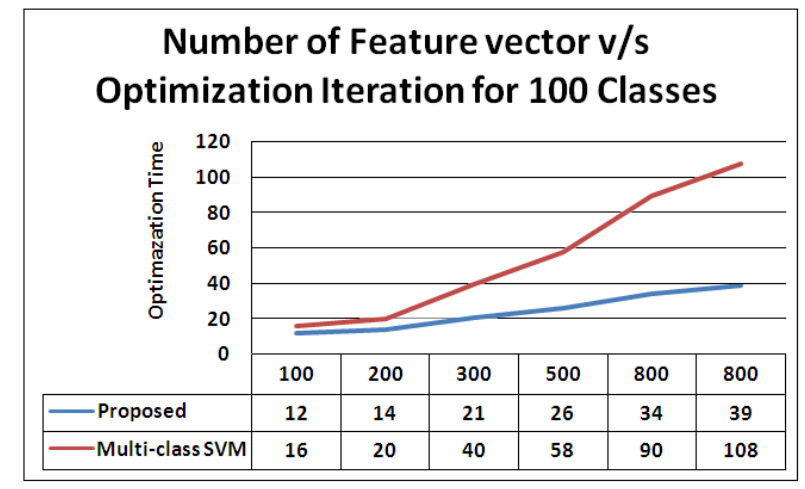

Fig 4: Plot of Number of feature vectors versus Optimization Iteration for 100 classes

Figure 4 shows the optimization time performance of the proposed system and Multi Class SVM technique. The graph justifies the fact that more features results in higher time complexity for the feature optimization and support vector generation. This time is also considered as the training time of the classifier. In IRIS recognition problem, though the training time plays lesser role in any biometric recognition problem, the classes are needed to be trained whenever a new class is added to the database. Therefore higher time in training and optimizing the database becomes significantly complex for frequently changing database. Proposed technique offers faster optimization which is one of the important aspects of a real time IRIS biometric based system. 


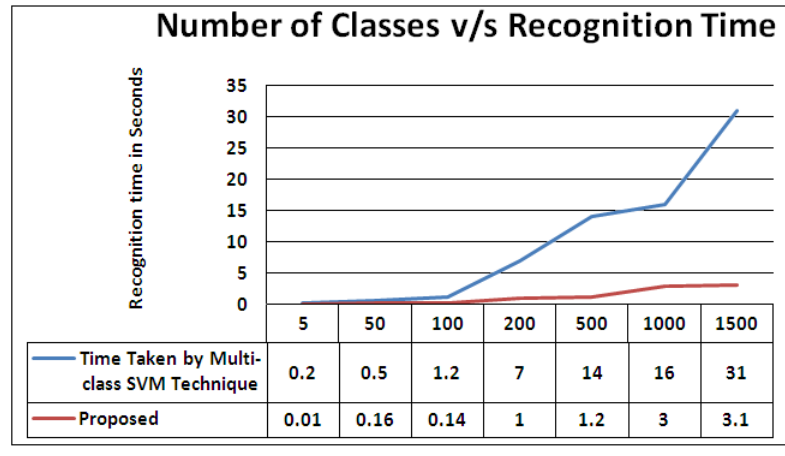

Fig 5: Plot of Number of classes versus Recognition Time

Recognition time is ideally the time required for a single test instance to be recognized as one of the trained instances. The performance in figure 5 shows that the proposed technique is a clear winner in terms of speed of the recognition. Coarse feature set presents several features that do not contribute for generating support vectors which are used for classifying the test features from the test IRIS instance. Quicker classification time is due to the fact that input or training vectors are preoptimized through dimensionality reduction technique. Therefore mapping the test features to the training domain is quicker as number of support vectors is less in the case of proposed system.

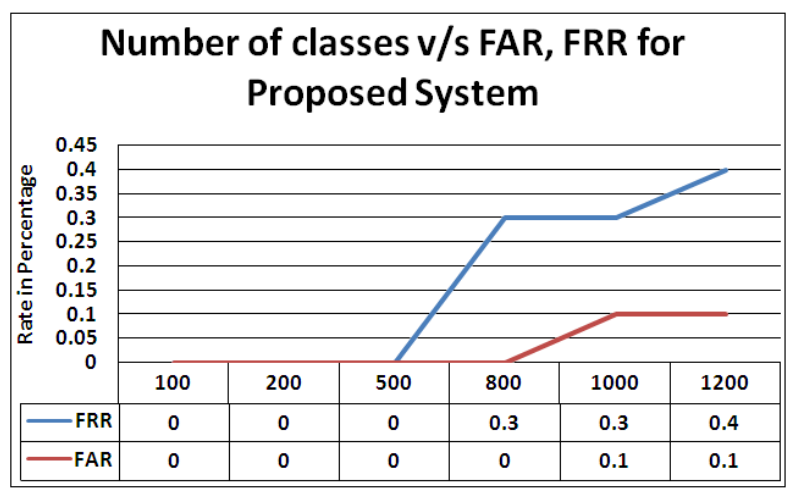

Fig 6: Plot of Number of classes versus FAR, FRR for proposed system

FAR and FRR analysis are most important part of any biometric system. For an ideal biometric system both must be close to zero and FRR must be higher than FAR. The performance result in figure 6 shows that FAR of the proposed system is significantly low with only one instance out of 1000 test instances being falsely accepted. False rejection rate can initiate a re-authentication process and is slightly higher than FAR which is again another desirable feature of a biometric system.

\section{CONCLUSION}

There are several IRIS recognition solutions being proposed over the years. These solutions are generally divided into two types: template based matching and classification based recognition. Several researchers have proved that classifier based technique produces better result than the template matching techniques in terms of accuracy. But complexity of the kernel increases with increase in number of classes and hence features. Hence kernel based classifiers are better used by first optimizing the features using a optimization technique like Quadratic programming based optimization. But the classifier fails to optimize the features for larger dataset to avoid drop in accuracy. Therefore a suitable dimensionality reduction technique is well suited to reduce the dimensions and hence the size of the feature vectors and followed by classifying the vectors using kernel based classifier like SVM. In this work we have proposed a Non linear dimensionality reduction technique with KPCA which is applied on the conventional feature set extracted from two dimensional Gabor filtering as has been widely used over past two decades since Daugman had first proposed it. Therefore the technique does not require any alternative feature selection method and uses the existing one for scalability and adoptability. Results shows that the overall accuracy of the system is near $100 \%$ ( $99.6 \%$ on CASIA IRIS database and $100 \%$ on UBIRIS database. The performance is better in terms of recognition rate and recognition time than Multi Class SVM based Technique. The technique can be further improved by analyzing the behavior of linear dimensionality reduction technique like PCA and mixed dimensionality reduction techniques with combined distance based classifier like KNN and kernel based classifier like SVM.

\section{REFERENCES}

[1] John G. Daugman, "High Confidence Visual Recognition of Persons by a Test of Statistical Independence", IEEE Transactions on Pattern Analysis and Machine Intelligence, Vol. 15, No.11, pp. 1148-1161, November 1993.

[2] John Daugman, "New Methods in Iris Recognition", IEEE Transactions on Systems, Man and Cybernetics Part B: Cybernetics, Vol. 37, No. 5, pp. 1167 - 1175, October 2007.

[3] John Daugman, "How Iris Recognition Works", IEEE Transactions on Circuits and Systems for Video Technology, Vol. 14, No. 1, pp. 21 - 30, January 2004.

[4] Amir Azizi and Hamid Reza Pourreza, "Efficient IRIS Recognition Through Improvement of Feature Extraction and Subset Selection", International Journal of Computer Science and Information Security, Vol. 2, No.1, June 2009.

[5] Kaushik Roy, Prabir Bhattacharya and Ramesh Chandra Debnath, "Multi-Class SVM Based Iris Recognition", 10th International Conference on Computer and information technology, vol. no., pp.1-6, 27-29 Dec. 2007.

[6] J. Li, D. Tao, W. Hu, and X. Li, "Kernel principle component analysis in pixels clustering," in Proc. IEEE/WIC/ACM Int. Conf. Web Intell., Sep. 2005, pp. 786-789.

[7] Y. Xu, C.-L. Lin and W. Zhao, Producing computationlly efficient KPCA-based Feature Extraction for Classification Problems, Electronics Letters 46(6) (2010).

[8] S. Lespinats, M. Verleysen, A. Giron, and G. Fertil, "DD-HDS: A method for visualization and exploration of high-dimensional data," IEEE Trans. Neural Netw., vol. 18, no. 5, pp. 1265-1279, Sep. 2007.

[9] Sang-Hyeun Park and Johannes F"urnkranz, "Efficient Pairwise Classifciation" Proceedings of 18th European Conference on Machine Learning (ECML-07), pp. 658665, Warsaw, Poland, 2007.

[10] Zheng Tao, Xie Mei , "Kernel Cluster and SVMs-Based Algorithm for Iris Rough Classification in Massive Databases", International Symposium on Computational 
Intelligence and Design, Vol. No.1, pp. 282-285, 17-18 October 2008.

[11] Huan-Jun Liu, Yao-Nan Wang, Xiao-Fen Lu, “A Method to Choose Kernel Function and its Parameters for Support Vector Machines", Proceedings of the Fourth International Conference on Machine Learning and Cybernetics, vol.7, no., pp. 4277-4280, August 2005.

[12] Proença, Hugo and Alexandre, Luís A., "UBIRIS: A noisy iris image database", Proceed. of ICIAP 2005 Intern. Confer. on Image Analysis and Processing, vol. 1, pp. 970-977, 2005

[13] Carlos Gershenson, "Artificial Neural Networks for Beginners". Cited as: arXiv:cs/0308031v1, 20 Aug 2003.
[14] C. T. Sun and J. S. Jang, "A neuro-fuzzy classifier and its applications," in Proc. IEEE Int. Conf. Fuzzy Syst., San Francisco, CA, Mar. 1993, vol. I, pp. 94-98.

[15] L. Shen, L. Bai, Gabor feature based face recognition using kernel methods, in: Proceedings of the Sixth IEEE International Conference on Automatic Face and Gesture Recognition (FGR'04), 2004.

[16] V.V.Satyanarayana Tallapragada, Dr. E.G.Rajan, "A Fast IRIS Recognition Technique Based on Dimensionality Optimization and Multidomain Feature Normalization", CiiT International Journal of Artificial Intelligent Systems and Machine Learning, Vol. 3, No.11, pp. 668676, October 2011.

[17] CASIA iris image database, available from http://www.cbsr.ia.ac.cn/IrisDatabase.htm 